\title{
Breaking down barriers: improving outcomes for teenagers and young adults with germ cell tumours
}

\author{
Matthew J. Murray • Lorna A. Fern • \\ Daniel P. Stark · Tim O. Eden · James C. Nicholson
}

Received: 9 October 2009/ Accepted: 13 October 2009/Published online: 4 November 2009

(C) Springer-Verlag 2009

\section{Background}

Teenagers and young adults (TYA) with cancer have complex and evolving needs which are unique to this patient group. The TYA age-group have outcomes that are not improving over time, lagging behind both adult and children's cancers in their rate of improvement in recent years [1]. This is closely associated with disappointing recruitment rates into clinical trials [2-7] for many different reasons [8, 9]. The poor recruitment of TYA patients into clinical trials may delay any improvements in survival [2] and in many TYA cancers, overall survival is worse than in children. In some cancers there is now evidence of superior survival for TYAs treated on paediatric rather than adult treatment protocols and the reasons for this require resolution $[3,10]$. Historically, however,

M. J. Murray ( $\square)$

MRC Cancer Cell Unit, Hutchison/MRC Research Centre,

Hills Road, Cambridge CB2 0XZ, UK

e-mail:mjm16@cam.ac.uk

\section{A. Fern}

National Cancer Research Institute, Teenage and Young Adult Clinical Studies Development Group (NCRI TYA CSDG),

University College Hospital, London, UK

D. P. Stark

St James's University Hospital, Leeds, UK

T. O. Eden

Young Oncology Unit, Manchester, UK

M. J. Murray · J. C. Nicholson

Addenbrooke's Hospital, Cambridge, UK

M. J. Murray · D. P. Stark · J. C. Nicholson

Children's Cancer and Leukaemia Group (CCLG)

GCT Working Group, Leicester, UK
TYAs have been neglected; treated by either paediatric or adult services as an 'addendum', based upon arbitrary, locally defined age-based cut-offs, irrespective of cancer epidemiology or patients' needs [5]. There is an urgent requirement to break down the historical barriers between adult and paediatric oncology approaches to patient management in order to optimize outcomes for this group of patients.

Germ cell tumours (GCTs) are heterogeneous tumours that are all believed to arise from a common precursor, the totipotent primordial germ cell [11], and are unique in occurring throughout life from the neonatal period into late adulthood. Their incidence rises through adolescence, peaking in early adulthood [12]. As current treatment strategies are successful in curing the majority of GCTs, current research includes integrating clinical and biological factors to improve prediction of outcome. This will allow subsequent stratification of patients to treatment regimens, with the aim of maintaining survival while sparing acute and late toxicity. Since predictive factors are likely to be found in the biological behaviour of these tumours, it is essential to increase the rates of entry into clinical trials incorporating biological studies, if we are to improve the outcome for TYA patients with GCTs. Decisions about patient management, however, are not currently influenced by biological differences across the age spectrum. Dedicated TYA multidisciplinary meetings attended by both paediatric and adult teams, as is beginning to be standard practice in the UK, may help to overcome such barriers, as these consider the biology of this disease.

\section{Epidemiology and incidence}

The incidence of GCTs increases rapidly in the TYA age range and this is thought to be associated with the altered 
Fig. 1 Incidence rates of germ cell tumours by anatomical site, per million person years at risk, for patients aged 0-79 years in England, 1995-2003. Each column on the graph represents a 5-year age range. (With thanks to Dr Robert Alston and Professor Jillian Birch, CRUK Paediatric and Familial Cancer Research Group, University of Manchester, UK)
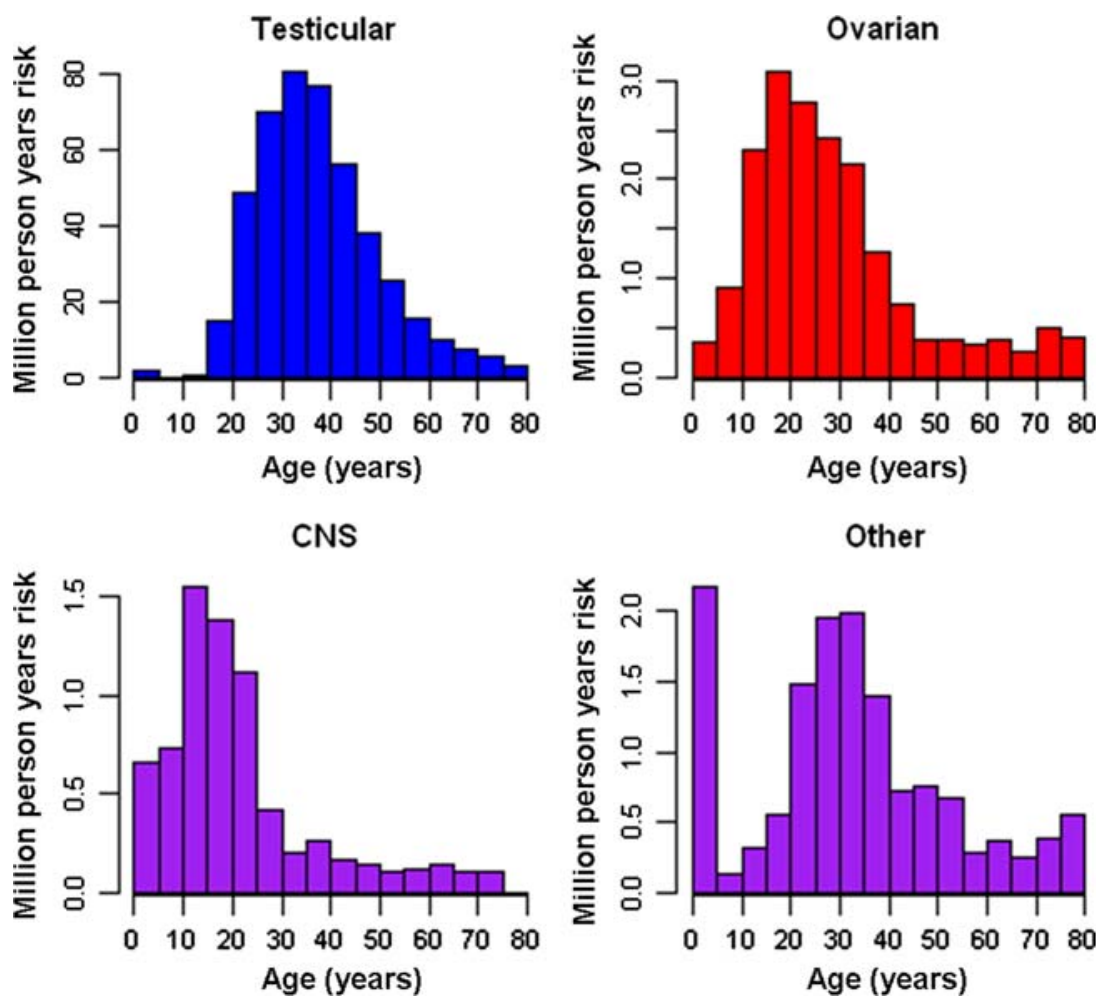

hormonal milieu at this time (Fig. 1). GCTs are the second most common individual malignancy in this age-group, representing over $13 \%$ of all cancers, with more than 250 patients diagnosed per year in England alone [13]. The incidence in males aged 13-24 years is 57.9 cases per million person years at risk, although rates in females are more modest at 7.0 cases per million person years. There has been a $2.9 \%$ annual increase in the incidence of GCTs in the TYA age-group in England but the aetiological factors responsible for this remain to be elucidated [14]. Overall incidence is much lower in late adulthood (14 per million population) and in young children (4 per million population) [15].

\section{Clinical and biological risk stratification}

At present, risk stratification for treatment is still based broadly on the clinical TNM classification, with the tumour markers alpha-fetoprotein and beta human choriogonadotrophin an additional factor in certain classifications. This is based upon substantial collaborative efforts in adult [16] and children's [17] oncology, but little work between the groups to allow focus on TYA patients. Differences remain between paediatric and adult stratification. Although the International Germ Cell Classification Consensus Group (IGCCCG) tumour marker criteria (developed for adult males with metastatic malignant GCTs) [16] were recently applied and shown to be prognostic for paediatric patients also, this produced a different stratification to that used in the Children's Oncology Group (COG) system in the US [18]. Development of a reliable classification applicable in children, TYAs and older adults and including an understanding of biology in this age-range would clearly facilitate the conduct of studies across this group. There are currently no molecular biological markers employed for risk stratification in any GCT treatment protocol. A key challenge will be the identification of predictive clinical markers that may be incorporated into such a system, in particular the ability to identify poor risk patients who are at increased risk of relapse. Due to the excellent prognosis for GCTs as a whole, with a greater than 90\% 5-year overall survival, such events are rare. Conversely, we need to identify those patients at very low risk of relapse in whom less toxic chemotherapy may be delivered whilst still maintaining survival rates. International co-operation will be required to enable such identification, both retrospectively in pooling pre-existing study and trial data but also prospectively in identifying a common data set which may easily be compared.

\section{Current treatment strategies}

Most clinicians employ platinum-based chemotherapeutic schedules to treat GCTs. Adult oncologists utilize 
bleomycin, etoposide and cisplatin ('BEP' or 'PEB') in advanced disease with overall survival rates well in excess of $90 \%$ [19, 20]. Late effects of chemotherapy include secondary malignancy (etoposide) in addition to pulmonary toxicity (bleomycin), ototoxicity, nephrotoxicity and subfertility (due to platinum agents; particularly cisplatin). Initially, paediatric patients were also treated on adult type cisplatin-based schedules, but more recently in some groups these have evolved, mainly in response to concerns about toxicity of cisplatin regimens when applied to children. Substitution of carboplatin for cisplatin in combination with etoposide and bleomycin ('JEB') has been shown to result in comparable survival for all tumour stages in children [17, 21].

There is a long history of progressive collaborative trial design in adult GCT management, providing an excellent model of a curable cancer in adults and including studies on the evaluation of late effects of curative treatment $[22,23]$. Attempts to reduce cisplatin toxicity in adults by substituting with carboplatin have resulted in an increased risk of relapse [24]. Such studies have demanded the use of lower platinum doses than have been tested in children because of the poorer tolerance of myelosuppression in older adults. With regards to bleomycin, inferior survival is observed with complete omission of this agent from adult protocols for certain GCT subtypes [25], but there is little data examining minimal effective dose or dose intensity of bleomycin, despite toxicity being related to total dose received [26]. Modification of the standard BEP regimes by reducing bleomycin dosage and frequency has been employed in adolescents [27], although achieving adequate statistical power in these studies with small numbers of events is difficult without wider collaboration. Bleomycin has been avoided entirely in some groups by employing a three drug regimen with cisplatin, ifosfamide and etoposide but again with concomitantly greater myelosuppression [28]. Young adults with leukaemia treated on a paediatric protocol had only marginally greater myelosuppression than children and adolescents when provided with the required level of protocol-defined supportive care, without affecting outcome [29], but the balance between different patterns of toxicity, acute treatment tolerance and later outcomes remains untested in TYAs with GCTs. At present, the needs of TYAs are not met by current studies which, although well-designed, have their primary focus on either younger children or older adults. In the future, clinical trials for those TYA and adult patients who are reliably predicted to be at low risk of relapse may include randomizations between standard (cisplatin-based) treatment and either carboplatin-based [30] or low-dose bleomycin protocols with the aim of maintaining excellent cure rates whilst minimising late effects. Conversely, TYA patients identified as high risk for relapse might receive experimental treatments, specifically derived for their needs and treatment tolerance.

\section{Clinical trials}

Through the work of the UK National Cancer Research Institute TYA Clinical Studies Development Group (NCRI TYA CSDG), we have detailed UK clinical trial data [4], which appears to reflect findings from the US. Despite the high incidence of GCTs in TYAs, the rate of trial recruitment in England from 2005 to 2007 for male GCT patients aged 15-24 years was $2.1 \%$ (8 of approximately 378 newly diagnosed patients) [4], compared with $42.9 \%$ for male patients aged $0-15$ years during the same period (6 out of 14 patients) [4]. Furthermore, there are currently no open trials for female GCT patients over the age of 18 years in the UK. These international observations of lower trial recruitment in TYA are multi-factorial [4], but seem unlikely to improve without collaboration between the groups designing trials and managing TYA patients. Poor recruitment to trials and lack of trial availability is a major issue that needs to be addressed if we are to make progress in our clinical and biological understanding of GCTs in TYAs, and subsequently improve outcomes.

\section{Biological research}

Tumour biology can form a common ground in building such collaborative working, allowing shared research objectives and productivity, and forming the basis of stratified TYAspecific trials. There has been much research into adult testicular GCT biology but little has been published on ovarian or paediatric [31-34] GCTs until recently. Gain of chromosome $12 \mathrm{p}$ is almost universal in adult GCTs but also occurs in a significant proportion of childhood cases [33]. There are currently no systematic research studies on the biology of GCTs in the TYA group. Clear differences have been identified in mRNA expression profiles between paediatric and adult GCT cases [32]. Limited access to biological tissue from adolescents meant that only two of the 49 samples $(4.1 \%)$ in this study were from patients aged 1524 years. Very recently, an mRNA gene expression signature has been identified and subsequently validated that predicts outcome in adult men with GCTs [35], independent of the IGCCCG risk classification. It remains to be seen whether this signature will be predictive in a TYA population. With the high incidence rates of GCTs in this age-group, potential for trial recruitment and lack of pre-existing comorbidity precluding trial entry, the TYA group may represent the very cohort best able to further our understanding and management of GCTs in general. Furthermore, in the 
UK, an effective tumour banking model has been established by the CCLG for collecting snap-frozen tissue. Clinicians and researchers involved in the management of TYAs should follow this lead, which will enhance the size, quality and statistical power of research studies undertaken.

\section{Consensus meeting}

The UK-based Teenage Cancer Trust (TCT) convened a consensus meeting in London in 2008 as a first step towards active collaboration between paediatric and adult GCT groups, in order to breakdown the conventional age-based barriers described and to achieve consensus to improve outcomes for TYAs with GCTs. This meeting brought together internationally recognized groups from both adult and paediatric practice, including clinicians, scientists and epidemiologists.

Clinical collaboration was established, and agreement was reached to combine international data sets across the TYA age-range. These will focus particularly on outcome in post-pubertal gonadal tumours, to enable the identification of high- and low-risk patients. For prospective collection of data, a common data set using a standardized international proforma is being explored. Furthermore, protocols in which biological tissue is collected as an integral component of the study should be actively encouraged in the future. This would allow for collection of snap-frozen tumour tissue wherever possible at diagnosis, even in trials without an active biological study embedded. At present, with our current lack of sufficient clinical and biological risk factors in TYA patients with GCTs, it was felt that new national or international clinical trials would be premature. However, ongoing laboratory exploration of the cisplatin sensitivity of tumours should continue [36], along with its relationship to tumour biology and clinical outcome. Furthermore, if the retrospective and prospective data analysis allows for definition of high- and low-risk groups then suitable trials could be established, with tumour biology (rather than arbitrary age cut-offs) as the criterion by which trials are defined. The management of TYAs with cancer should follow their biological, clinical and wider risk and needs, rather than a historical professional delineation. This can only be achieved through collaboration. A second meeting is therefore currently being planned to consolidate progress and explore areas for further collaboration.

\section{Conclusion}

The model we describe is one that may be reproduced to address common issues applicable to all TYAs as well as tumour-specific groups. GCTs are a particularly good model for collaboration between paediatric and adult oncology communities given the greatest incidence of these tumours in this age-group. Consensus meetings as described should help to breakdown conventional agebased barriers currently present in paediatric and adult cancer care. Outcomes for TYAs with GCTs and other tumour types will not improve unless such obstacles are overcome.

Acknowledgments We are most grateful to Micky and Julie Walker, for sponsoring the consensus meeting through the Katie Walker Cancer Trust, and to the TCT, for arranging the meeting. In addition to the participants of the meeting who contributed to the discussions, listed below, we are particularly grateful to Professor Jillian Birch and Dr Robert Alston (CRUK Paediatric and Familial Cancer Research Group, University of Manchester) for the incidence data.

Conflict of interest statement None declared.

\section{Appendix}

Contributors to the consensus meeting and institutions represented

Karen Albritton, Dana-Farber Cancer Institute, Boston, USA; James Amatruda, UT Southwestern Medical Center, Dallas, USA; Sebastian Bauer, Westgerman Cancer Center, Essen, Germany; Jillian Birch, CRUK Research Group, University of Manchester, UK; Tim Eden, Young Oncology Unit, University of Manchester, UK; Lorna Fern, NCRI TYA CSDG, UCLH, UK; Lindsay Frazier, DanaFarber Cancer Institute, Boston, USA; Juliet Hale, Royal Victoria Infirmary, Newcastle, UK; Mike Leahy, Christie Hospital, Manchester, UK; Leendert Looijenga, Erasmus Medical Center, UMC, Rotterdam, Netherlands; Matthew Murray, MRC Cancer Cell Unit, Cambridge, UK; Craig Nichols, Providence Cancer Center, Portland, USA; James Nicholson, Addenbrooke's Hospital, Cambridge, UK; Tim Oliver, St Bartholomew's Hospital, London, UK; Tom Olson, Children's Healthcare of Atlanta, Atlanta, USA; Roger Palmer, MRC Cancer Cell Unit, Cambridge, UK; Gordon Rustin, Mount Vernon Hospital, Hertfordshire, UK; Dominik Schneider, Clinic of Paediatrics, Dortmund, Germany; Stefan Schönberger, University Children's Hospital, Düsseldorf, Germany; Janet Shipley, Institute of Cancer Research, Sutton, Surrey, UK; Sara Stoneham, UCLH, London, UK; Jeremy Whelan, NCRI TYA CSDG Chair, UCLH, UK; Jeff White, Beatson Oncology Centre, Glasgow, UK; Myrna Whiteson MBE, TCT Chairman, UK. 


\section{References}

1. Bleyer A, Barr R, Hayes-Lattin B, Thomas D, Ellis C, Anderson B (2008) The distinctive biology of cancer in adolescents and young adults. Nat Rev Cancer 8:288-298

2. Bleyer A, Montello M, Budd T, Saxman S (2005) National survival trends of young adults with sarcoma: lack of progress is associated with lack of clinical trial participation. Cancer 103:1891-1897

3. Bleyer WA (2002) Cancer in older adolescents and young adults: epidemiology, diagnosis, treatment, survival, and importance of clinical trials. Med Pediatr Oncol 38:1-10

4. Fern L, Davies S, Eden T, Feltbower R, Grant R, Hawkins M et al (2008) Rates of inclusion of teenagers and young adults in England into National Cancer Research Network clinical trials: report from the National Cancer Research Institute (NCRI) Teenage and Young Adult Clinical Studies Development Group. Br J Cancer 99:1967-1974

5. Fernandez CV, Barr RD (2006) Adolescents and young adults with cancer: an orphaned population. Paediatr Child Health 11:103-106

6. Ferrari A, Bleyer A (2007) Participation of adolescents with cancer in clinical trials. Cancer Treat Rev 33:603-608

7. Whelan JS, Fern LA (2008) Poor accrual of teenagers and young adults into clinical trials in the UK. Lancet Oncol 9:306-307

8. Burke ME, Albritton K, Marina N (2007) Challenges in the recruitment of adolescents and young adults to cancer clinical trials. Cancer 110:2385-2393

9. Ferrari A, Montello M, Budd T, Bleyer A (2008) The challenges of clinical trials for adolescents and young adults with cancer. Pediatr Blood Cancer 50:1101-1104

10. Hallbook H, Gustafsson G, Smedmyr B, Soderhall S, Heyman M (2006) Treatment outcome in young adults and children $>10$ years of age with acute lymphoblastic leukemia in Sweden: a comparison between a pediatric protocol and an adult protocol. Cancer 107:1551-1561

11. Teilum G (1965) Classification of endodermal sinus tumour (mesoblatoma vitellinum) and so-called "embryonal carcinoma" of the ovary. Acta Pathol Microbiol Scand 64:407-429

12. Cancer Research UK (CRUK) (2009) Testicular cancer incidence statistics. http://info.cancerresearchuk.org/cancerstats/types/ testis/incidence/\#age.q Accessed 9 Oct 2009

13. Birch JM, Alston RD, Kelsey AM, Quinn MJ, Babb P, McNally RJ (2002) Classification and incidence of cancers in adolescents and young adults in England 1979-1997. Br J Cancer 87: 1267-1274

14. Alston RD, Geraci M, Eden TO, Moran A, Rowan S, Birch JM (2008) Changes in cancer incidence in teenagers and young adults (ages 13 to 24 years) in England 1979-2003. Cancer 113: 2807-2815

15. Office of National Statistics (ONS) Cancer statistics registrations (2008) http://www.statistics.gov.uk/downloads/theme_health/ MB1-37/MB1_37_2006.pdf. Accessed 9 Oct 2009

16. Group International Germ Cell Cancer Collaborative (1997) International Germ Cell Consensus Classification: a prognostic factor-based staging system for metastatic germ cell cancers. J Clin Oncol 15:594-603

17. Mann JR, Raafat F, Robinson K, Imeson J, Gornall P, Sokal M et al (2000) The United Kingdom Children's Cancer Study Group's second germ cell tumor study: carboplatin, etoposide, and bleomycin are effective treatment for children with malignant extracranial germ cell tumors, with acceptable toxicity. J Clin Oncol 18:3809-3818

18. Frazier AL, Rumcheva P, Olson T, Giller R, Cushing B, Cullen J et al (2008) Application of the adult international germ cell classification system to pediatric malignant non-seminomatous germ cell tumors: a report from the Children's Oncology Group. Pediatr Blood Cancer 50:746-751

19. Krege S, Beyer J, Souchon R, Albers P, Albrecht W, Algaba F et al (2008) European consensus conference on diagnosis and treatment of germ cell cancer: a report of the second meeting of the European Germ Cell Cancer Consensus group (EGCCCG): part I. Eur Urol 53:478-496

20. Krege S, Beyer J, Souchon R, Albers P, Albrecht W, Algaba F et al (2008) European consensus conference on diagnosis and treatment of germ cell cancer: a report of the second meeting of the European Germ Cell Cancer Consensus Group (EGCCCG): part II. Eur Urol 53:497-513

21. Stern JW, Bunin N (2002) Prospective study of carboplatin-based chemotherapy for pediatric germ cell tumors. Med Pediatr Oncol 39:163-167

22. de Wit R, Fizazi K (2006) Controversies in the management of clinical stage I testis cancer. J Clin Oncol 24:5482-5492

23. Kondagunta GV, Motzer RJ (2006) Chemotherapy for advanced germ cell tumors. J Clin Oncol 24:5493-5502

24. Horwich A, Sleijfer DT, Fossa SD, Kaye SB, Oliver RT, Cullen MH et al (1997) Randomized trial of bleomycin, etoposide, and cisplatin compared with bleomycin, etoposide, and carboplatin in good-prognosis metastatic nonseminomatous germ cell cancer: a Multiinstitutional Medical Research Council/European Organization for Research and Treatment of Cancer Trial. J Clin Oncol 15:1844-1852

25. de Wit R, Stoter G, Kaye SB, Sleijfer DT, Jones WG, ten Bokkel Huinink WW et al (1997) Importance of bleomycin in combination chemotherapy for good-prognosis testicular nonseminoma: a randomized study of the European Organization for Research and Treatment of Cancer Genitourinary Tract Cancer Cooperative Group. J Clin Oncol 15:1837-1843

26. O'Sullivan JM, Huddart RA, Norman AR, Nicholls J, Dearnaley DP, Horwich A (2003) Predicting the risk of bleomycin lung toxicity in patients with germ-cell tumours. Ann Oncol 14:9196

27. Rogers PC, Olson TA, Cullen JW, Billmire DF, Marina N, Rescorla F et al (2004) Treatment of children and adolescents with stage II testicular and stages I and II ovarian malignant germ cell tumors: a Pediatric Intergroup Study-Pediatric Oncology Group 9048 and Children's Cancer Group 8891. J Clin Oncol 22:3563-3569

28. Nichols CR, Catalano PJ, Crawford ED, Vogelzang NJ, Einhorn LH, Loehrer PJ (1998) Randomized comparison of cisplatin and etoposide and either bleomycin or ifosfamide in treatment of advanced disseminated germ cell tumors: an Eastern Cooperative Oncology Group, Southwest Oncology Group, and Cancer and Leukemia Group B Study. J Clin Oncol 16:1287-1293

29. Ribera JM, Oriol A, Sanz MA, Tormo M, Fernandez-Abellan P, del Potro E et al (2008) Comparison of the results of the treatment of adolescents and young adults with standard-risk acute lymphoblastic leukemia with the Programa Espanol de Tratamiento en Hematologia pediatric-based protocol ALL-96. J Clin Oncol 26:1843-1849

30. Tscherry G, Jacky E, Jost LM, Stahel RA (2000) Risk-adapted chemotherapy of germ cell tumors with carboplatin, etoposide and bleomycin for low-risk and cisplatin, etoposide and ifosfamide for high-risk patients. A single-center study. Oncology 59:110-117

31. Fritsch MK, Schneider DT, Schuster AE, Murdoch FE, Perlman EJ (2006) Activation of Wnt/beta-catenin signaling in distinct histologic subtypes of human germ cell tumors. Pediatr Dev Pathol 9:115-131

32. Palmer RD, Barbosa-Morais NL, Gooding EL, Muralidhar B, Thornton CM, Pett MR et al (2008) Pediatric malignant germ cell 
tumors show characteristic transcriptome profiles. Cancer Res 68:4239-4247

33. Palmer RD, Foster NA, Vowler SL, Roberts I, Thornton CM, Hale JP et al (2007) Malignant germ cell tumours of childhood: new associations of genomic imbalance. Br J Cancer 96:667-676

34. Schneider DT, Schuster AE, Fritsch MK, Calaminus G, Harms D, Gobel U et al (2001) Genetic analysis of childhood germ cell tumors with comparative genomic hybridization. Klin Padiatr 213:204-211
35. Korkola JE, Houldsworth J, Feldman DR, Olshen AB, Qin LX, Patil S et al (2009) Identification and validation of a gene expression signature that predicts outcome in adult men with germ cell tumors. J Clin Oncol. doi:10.1200/JCO.2008.20.0386

36. Mueller S, Schittenhelm M, Honecker F, Malenke E, Lauber K, Wesselborg S et al (2006) Cell-cycle progression and response of germ cell tumors to cisplatin in vitro. Int J Oncol 29:471-479 